\title{
3D printing in medicine: current applications and future directions
}

\author{
Zhonghua Sun \\ Discipline of Medical Radiation Sciences, School of Molecular and Life Sciences, Curtin University, Perth, Western Australia, Australia \\ Correspondence to: Professor Zhonghua Sun. Discipline of Medical Radiation Sciences, School of Molecular and Life Sciences, Curtin University, \\ GPO Box U1987, Perth, Western Australia 6845, Australia. Email: z.sun@curtin.edu.au.
}

Submitted Nov 30, 2018. Accepted for publication Dec 10, 2018.

doi: 10.21037/qims.2018.12.06

View this article at: http://dx.doi.org/10.21037/qims.2018.12.06

\section{Introduction}

Technical developments in medical imaging techniques have led to significant improvements in the diagnostic performance of less-invasive imaging modalities such as computed tomography (CT), magnetic resonance imaging (MRI), nuclear medicine and ultrasound. Quantitative analysis of these imaging modalities allows for detection and diagnosis of various diseases with high accuracy (1-10). Despite promising results available in the literature, traditional two-dimensional (2D) and three-dimensional (3D) visualization tools are still limited to a $2 \mathrm{D}$ screen, which affect realistic visualization of anatomical structures and pathologies of $3 \mathrm{D}$ datasets, and this is particularly apparent when dealing with complex pathologies. This has created potential opportunities for the use of $3 \mathrm{D}$ printing technique in medical applications.

$3 \mathrm{D}$ printing has shown increasing applications in the medical field over the last decades with reports covering different areas which range from its original applications in orthopedics to cardiovascular disease and tumor imaging (11-15). In 2016, the Special Interest Group (SIG) on 3D printing was established by the Radiological Society of North America (RSNA) to provide guidelines, recommendations and training sessions for the appropriate use of $3 \mathrm{D}$ printing in medical applications with the aim of providing better healthcare service to patients $(16,17)$. There is a growing body of evidence in the literature to show how 3D-printed models assist clinicians to better manage patients with improved clinical outcomes. This editorial mainly focuses on the rapidly expanding applications of $3 \mathrm{D}$ printing in medicine, in particular in congenital heart disease (CHD), vascular disease and tumors. Furthermore, an emerging area of using $3 \mathrm{D}$-printed models to develop optimal CT scanning protocols is also discussed, with future research directions highlighted.

\section{Current clinical applications}

Patient-specific 3D-printed models are most commonly generated by using patient's CT or MRI imaging data which undergo a series of image postprocessing and segmentation steps to extract the regions of interest (desired anatomical structures and pathologies) from volumetric data. $3 \mathrm{D}$-printed models have been shown to accurately replicate normal anatomical structures and pathologies (18-20). Clinical value of 3D-printed models in the craniomaxillofacial area has been confirmed since the late 1980s $(21,22)$. According to the appropriateness guidelines developed by the SIG, 3D-printed models have shown advantages and usefulness in patients with complex skull, facial and mandibular fractures, and temporomandibular joint disorders, benign and malignant tumors (16). This has been well supported by sufficient studies in the literature, thus it won't be covered in this editorial. Emerging and new applications are showing promise in cardiovascular disease, tumor imaging and optimal CT scanning protocols, hence, the following sections will focus on these areas.

\section{D-printed models in CHD}

Due to complex cardiac anatomy associated with CHD, 3D printing serves as a useful tool to improve the understanding of CHD. Studies support the clinical value of 3D-printed models in CHD, specifically with reported usefulness of $3 \mathrm{D}$ printing in pre-surgical planning and simulation of 
complex situations $(9,18,23-25)$. Although most of the current literature on $3 \mathrm{D}$ printing in CHD is dominated by case reports and case series, emerging evidence based on randomized controlled trials and multicenter studies further confirms its incremental value over traditional visualization tools (26-31).

Loke et al. studied how the 3D-printed heart models impacted on learning complex CHD such as tetralogy of Fallot (ToF) (28). They randomly assigned 35 second year pediatric residents to two groups with 17 using standard 2D method of depicting ToF and 18 using 3D-printed model of the cardiac defect. Both groups were asked to complete pre- and post-session questionnaires to determine their knowledge, learning satisfaction and self-efficacy ratings about cardiac defects. Their results showed that 3D-printed models enhanced residents' understanding and satisfaction during the teaching sessions when compared to $2 \mathrm{D}$ images, and increased their confidence in managing patients with ToF. Similar findings are reported by White et al. (29) who divided 60 pediatric residents into two groups, 29 participating in the control group and 31 in the intervention group. Both groups received the same 20-min lecture including 2D images of ventricular septal defect (VSD) or ToF, while the intervention group was given $3 \mathrm{D}$-printed models of normal hearts and each CHD. Although 3D-printed models did not add beneficial value for understanding simple CHD such as VSD, they significantly increased participant's confidence and knowledge in complex CHD such as ToF. This further confirms the advantages of 3D-printed physical models over traditional image visualizations for demonstrating complex cardiac lesions with a higher level of understanding.

Valverde $e t a l$. reported their multicenter study experience of using 3D-printed models of CHD and the corresponding impact on surgical planning and treatment (30). This study included 40 patients with complex CHD from ten international centers. 3D-printed models were generated using CT and MRI images with high accuracy of depicting anatomical structures (mean bias: $-0.27 \pm 0.73 \mathrm{~mm}$ ). $3 \mathrm{D}$-printed models did not lead to change in surgical decision in $52.5 \%$ cases, most likely due to simplicity of the CHD cases. However, in nearly half of the cases (48\%), the surgical approach was redefined by use of $3 \mathrm{D}$-printed models, highlighting the clinical value of $3 \mathrm{D}$-printed models in managing complex cases.

In a recent study, Ryan et al. reported their single center experience of using 3D-printed models in CHD (31). Over a duration of 3-year period, 928 cardiothoracic surgeries were performed at their pediatric hospital, of which 164 anatomical models were generated for different purposes, such as education, patient-doctor communication, presurgical intervention and planning. Seventy-nine 3D models were specially used in the surgical planning of CHD cases. This retrospective study demonstrates the integration of $3 \mathrm{D}$ printing technology into clinical practice and its impact on patient's care with reductions in the operation room and care length with assistance of $3 \mathrm{D}$-printed models. Furthermore, the 30-day readmission and 30-day mortality rates were lower with use of $3 \mathrm{D}$-printed models when compared to the standard of care, although this did not reach statistical significance. $3 \mathrm{D}$-printed models have been further confirmed to be an effective tool in surgical planning of complex CHD patients, although prospective multicenter trials are needed to validate these findings.

\section{D-printed models in cardiovascular disease}

Applications of 3D printing in other cardiovascular disease or cerebrovascular disease are mainly based on isolated case reports or case series (23,32-36). Systematic reviews of cardiovascular disease including CHD showed that 42-61\% of studies were based on case reports of 3D-printed heart or vascular models (23,32-34). In addition to its usefulness in replicating vascular diseases such as aortic/cerebral aneurysm and pre-surgical planning and simulations, 3D-printed models are also reported to guide stent graft placement and assess coronary lumen stenosis due to calcified plaques $(37,38)$.

Our experience shows that 3D-printed models accurately replicated aortic aneurysm and aortic dissection based on patient's CT imaging data, although some measurement differences at the aortic locations and true lumen were more than $1.0 \mathrm{~mm}(33,34)$. It is challenging to reproduce the intimal flap on 3D printing models due to very thin and fine structure, however, this was addressed by Hossien et al. (35). Authors created 3D-printed models of type A acute aortic dissection in three cases with aortic tear commencing in the ascending aorta with involvement of aortic branches to some extent. Solid aortic models were produced using polylactic acid materials with demonstration of true and false lumen in relation to the aortic branches (Figure 1). They also demonstrated the intimal flap which separates the true lumen from the false lumen in the $3 \mathrm{D}$-printed model (Figure 2). These models can be used to guide complex endovascular repair of aortic dissection, allow for surgical simulation and serve as a training tool for medical students and clinicians. 

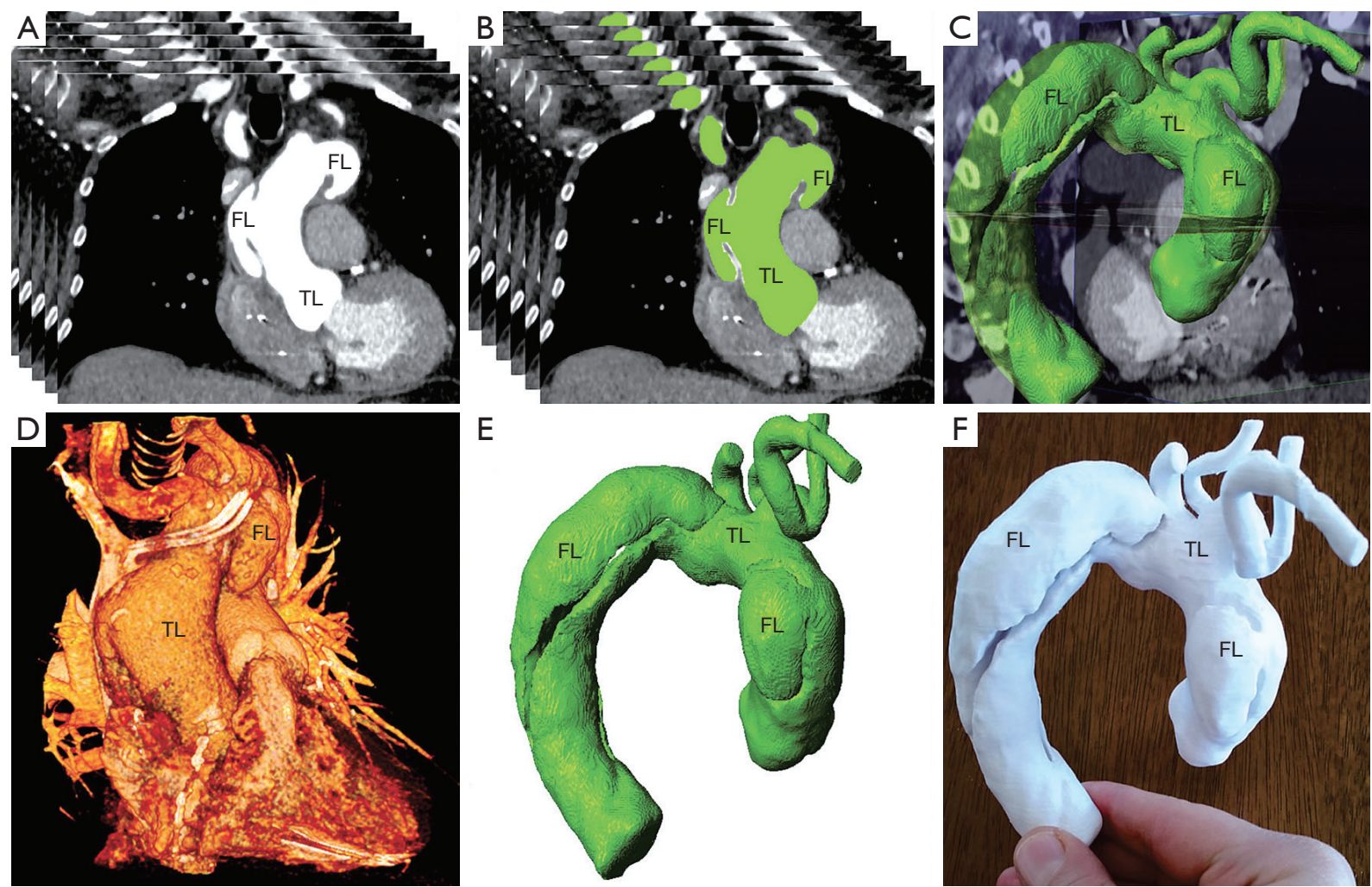

Figure 1 Three-dimensional (3D) reconstruction of the aortic dissection of a patient, where the aortic tear located in all segments of the aorta with flap propagated to all segments of the aorta. (A) Computed tomography scan of the chest showing complete dissected aorta; (B) thresholding of the dissected aorta; (C) integration of segmented aorta with 3D CT image; (D) 3D surface rendering of the aorta and surrounding structures; (E) 3D segmentation of the aorta; (F) 3D-printed model of the TAAD. TAAD, type A acute aortic dissection; TL, true lumen; FL, false lumen. Reprinted with permission from Hossien et al. (35).
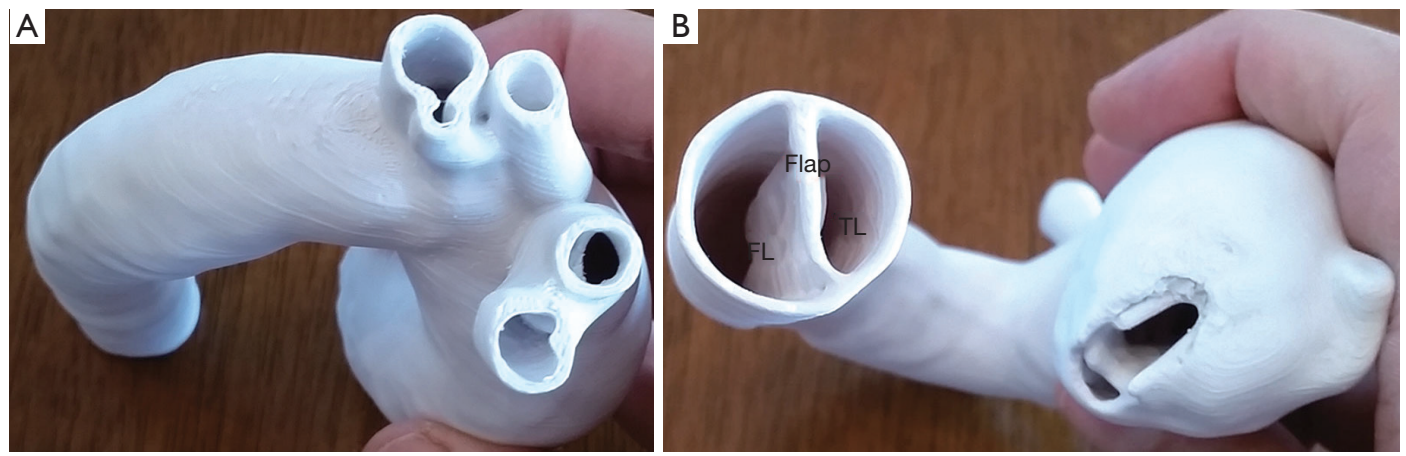

Figure 2 3D-printed model showing aortic branches and aortic dissection including intimal flap. (A,B) 3D printing of all components of TAAD (aortic wall, TL, FL and flap). TAAD, type A acute aortic dissection; TL, true lumen; FL, false lumen. Reprinted with permission from Hossien et al. (35).

Huang and colleagues in their case report further applied the $3 \mathrm{D}$-printed model to guide fenestrated stent grafting in a patient diagnosed with juxtarenal aortic aneurysm (37).
Different from other studies, they produced a 3D-printed skin template based on CT data with the aim of locating the fenestration position on the stent graft. The skin template 

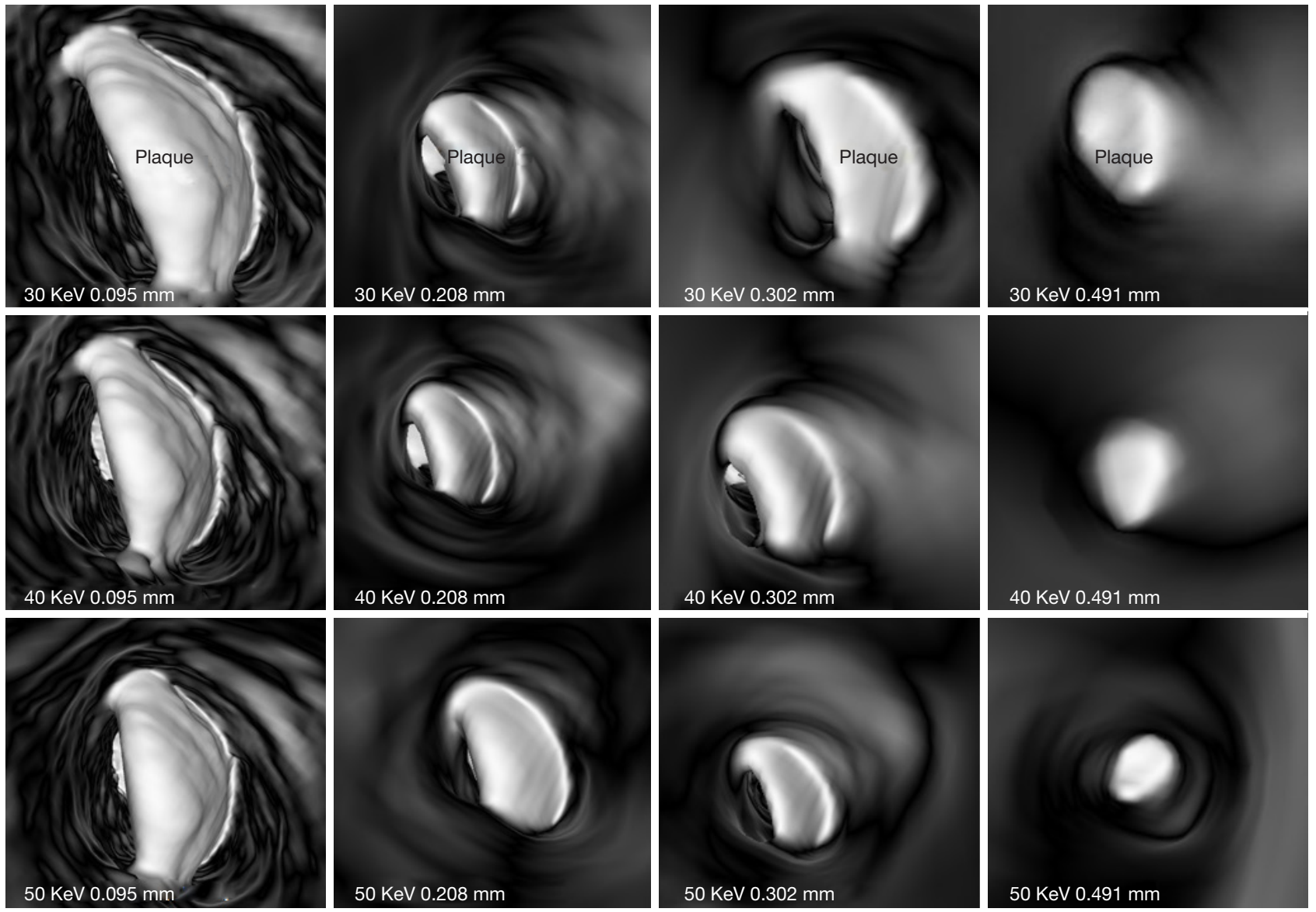

Figure 3 3D virtual intravascular endoscopy images of plaque at left circumflex acquired with different beam energies and slice thicknesses. The plaque became irregular when the slice thickness of $0.491 \mathrm{~mm}$ was used for image reconstruction. Arrows indicate simulated thrombus in the main pulmonary arteries. Reprinted with permission from Sun et al. (38).

was used to cover the stent graft prior to the surgical procedure for providing accurate location of fenestration holes on the stent graft. This represents a novel approach for improving fenestration accuracy on the stent graft, although further research on more cases is needed.

Our recent phantom experiments using high resolution synchrotron radiation have demonstrated the effect of spatial resolution on the visualization of coronary calcified plaques and associated lumen stenosis (38). Patient-specific coronary artery models were printed using soft and elastic TangoPlus material, while calcified plaques were printed with rigid VeroWhite material to represent calcification. These plaques were inserted into the three coronary models simulating $45-90 \%$ lumen stenosis with images acquired with high resolution synchrotron radiation $\mathrm{CT}$ resulting in slice thicknesses ranging from $0.095,0.208,0.302$ to
$0.491 \mathrm{~mm}$. Both 2D and 3D assessments showed that the high-resolution images (acquired with resolutions between 0.095 and $0.302 \mathrm{~mm}$ ) allow for accurate assessment of coronary plaques and lumen stenosis, while images acquired with a slice thickness of $0.491 \mathrm{~mm}$ result in significant overestimation of stenosis due to calcified plaques (Figure 3). High resolution or ultra-high-resolution CT indicates the research direction of improving diagnostic value of CT angiography in calcified plaques.

\section{D-printed models in tumors}

Imaging techniques play an important role in the diagnostic assessment of tumors with regard to size, location and extent of the tumors which are essential for surgical planning and decision making of tumor resection. Due to 

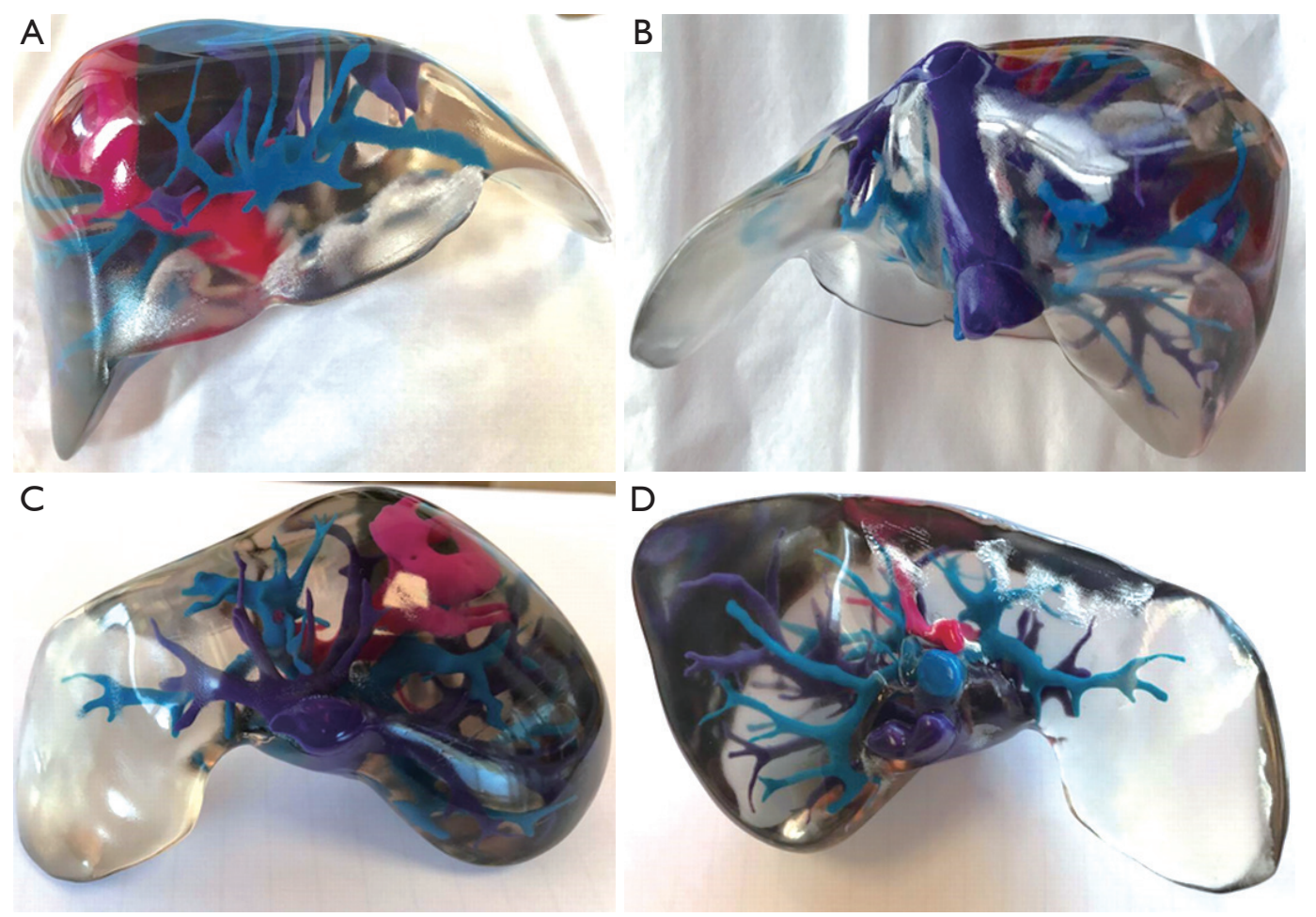

Figure 4 Anterior (A), posterior (B), superior (C), and inferior (D) views of the 3D-printed liver model generated from CT images, demonstrating the liver parenchyma (transparent), inferior vena cava and hepatic veins (purple), portal veins (blue), the tumor, and hepatic arterial supply (pink). 3D, three-dimensional; CT, computed tomography. Reprinted with permission from Perica and Sun (42).

complexity of relationships between tumor and surrounding structures, $3 \mathrm{D}$ printing technique has shown great value in assisting tumor pre-surgical planning and simulation with studies documenting different types of tumors that involved brain, liver, kidneys and heart (39-45).

Of these applications, 3D printing has been increasingly used in the pre-surgical planning and simulation of hepatic and renal malignant tumors. A recent systematic review has analyzed 19 studies reporting the clinical application of 3D-printed liver models (39). Despite most of the studies being case reports in the review, qualitative and quantitative results showed the usefulness of the $3 \mathrm{D}$-printed models in the preoperative planning and simulation of surgical procedures of liver lesions, as well as in medical education and training (Figure 4). 3D-printed models are shown to accurately detect hepatic structures and tumors with high accuracy when compared to original CT images, according to a recent study with analysis of 15 patient-specific $3 \mathrm{D}$ models (20).

Similarly, another systematic review has analyzed 15 studies reporting the $3 \mathrm{D}$-printed kidney models (15). Of these studies, more than half of them (53\%) reported quantitative findings of $3 \mathrm{D}$-printed model accuracy in displaying renal structures or renal tumors, or reduction in intraoperative examination time, while the remaining seven studies reported qualitative analysis of 3D-printed kidney models in improving patient's understanding of normal anatomy and pathology, and clinical value of $3 \mathrm{D}$-printed in models in pre-surgical planning or simulation of renal procedures and reduction of complications associated with operations. Despite these promising results, clinical trials with inclusion of a large cohort of data at multiple clinical sites are needed.

\section{D-printed models in optimization of CT scanning protocols}

An emerging research direction in using 3D-printed models is to develop optimal scanning protocols with low radiation dose and acceptable image quality (46). Traditionally, anthropomorphic phantoms are used to optimize imaging protocols, however, these phantoms only represent average 

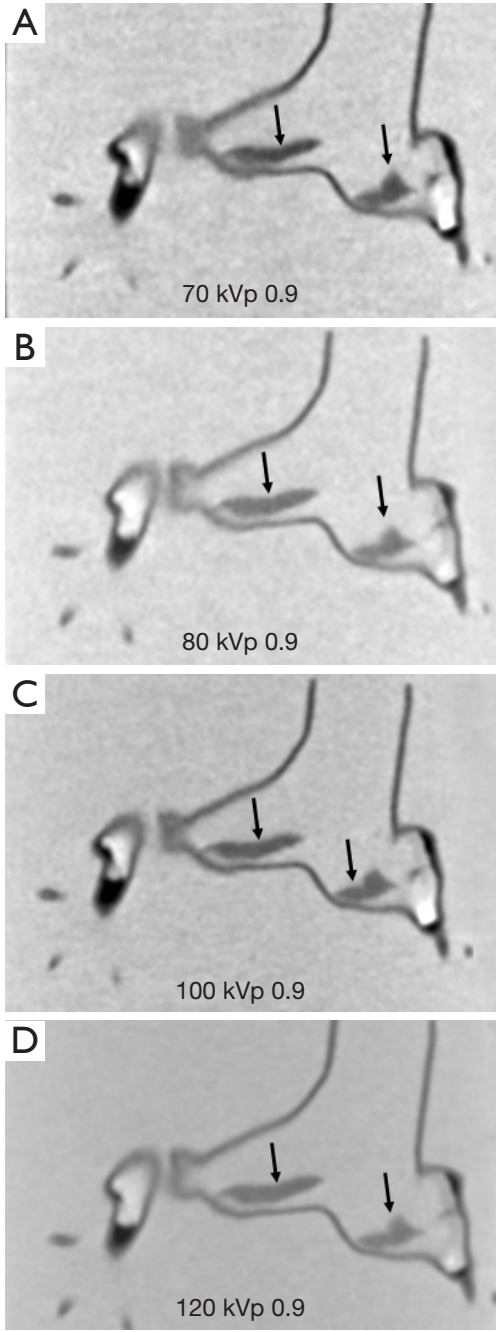
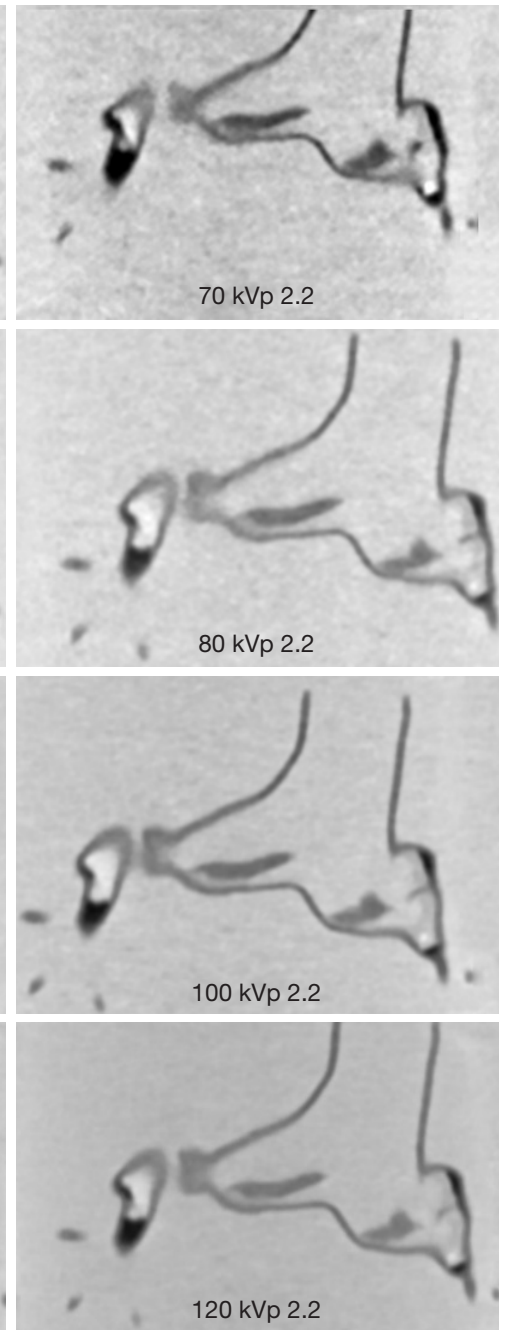
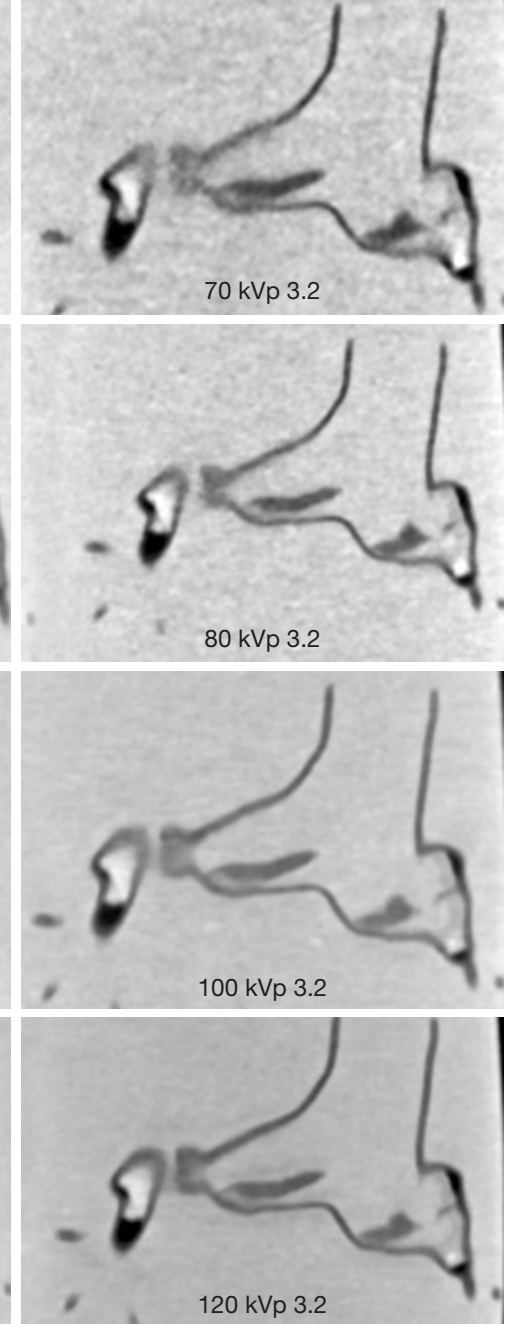

Figure 5 CTPA protocols with use of different $\mathrm{kVp}$ and pitch values. (A,B) When pitch was increased to 3.2, image noise was increased with 70 and $80 \mathrm{kV}$ p protocols; (C,D) in contrast, no significant change of image quality was noted with 100 and $120 \mathrm{kVp}$ protocols, regardless of pitch values. CTPA, computed tomography pulmonary angiography. Reprinted with permission from Aldosari et al. (48).

adults or pediatrics. Patient-specific 3D-printed models based on patient's imaging data offer realistic models with a high accuracy in replicating anatomy and pathology, thus serving as a reliable tool to optimize CT protocols.

Currently, 3D-printed heart and pulmonary artery models have been used to test different CT scanning protocols for dose optimization (47-49). The 3D-printed cardiac insert phantom developed by Abdullah et al. represents a novel approach for optimizing cardiac CT protocols (48), while 3D-printed pulmonary artery model by Aldosari et al. indicates another new approach for optimizing CT pulmonary angiography protocols with simulation of main and peripheral pulmonary embolism (Figure 5). Significant dose reduction up to $80 \%$ can be achieved with resulting diagnostic images of detecting pulmonary embolism at the main and peripheral pulmonary arteries, thus highlighting the feasibility of using 3D-printed pulmonary model for developing low-dose CT protocols $(48,49)$.

\section{Summary and future research directions}

$3 \mathrm{D}$-printed models have been used in many medical areas ranging from accurate replication of anatomy and pathology to assist pre-surgical planning and simulation of complex surgical or interventional procedures, serve as a useful tool for education of medical students and patients, 
and improve doctor-patient communication. Furthermore, patient-specific 3D-printed models can be used as a costeffective tool to develop optimal CT scanning protocols. Current research should go beyond investigation of the model accuracy to focus more on clinical trials regarding the impact of $3 \mathrm{D}$ printing on clinical decision-making and patient's outcomes. Prospective studies with inclusion of more cases at multi-center sites are desirable to validate these findings. With further technical improvements in $3 \mathrm{D}$ printing techniques and reductions in printing cost and image post-processing time, $3 \mathrm{D}$ printing will be incorporated into routine clinical diagnosis in the near future.

\section{Acknowledgements}

Funding: This work was supported by National Heart Foundation of Australia (No. 101843).

\section{Footnote}

Conflicts of Interest: The author has no conflicts of interest to declare.

\section{References}

1. Loughborough WW, Yeong M, Hamilton M, Manghat $\mathrm{N}$. Computed tomography in congenital heart disease: how generic principles can be applied to create bespoke protocols in the Fontan circuit. Quant Imaging Med Surg 2017;7:79-87.

2. Liu D, Fan Z, Li Y, Zhang N, Sun Z, An J, Stalder AF, Greiser A, Liu J. Quantitative study of abdominal blood flow patterns in patients with aortic dissection by 4-dimensional MRI. Sci Rep 2018;8:9111.

3. Wang R, Liu X, Schoepf UJ, van Assen M, Alimohamed I, Griffith LP, Luo T, Sun Z, Fan Z, Xu L. Extracellular volume quantification using dual-energy $\mathrm{CT}$ in patients with heart failure: comparison with 3T cardiac MR. Int J Cardiol 2018;268:236-40.

4. Hoey ET, Shahid M, Watkin RW. Computed tomography and magnetic resonance imaging evaluation of pericardial disease. Quant Imaging Med Surg 2016;6:274-84.

5. D'Errico L, Salituri F, Ciardetti M, Favilla R, Mazzarisi A, Coppini G, Bartolozzi C, Marraccini P. Quantitative analysis of epicardial fat volume: effects of scanning protocol and reproducibility of measurements in noncontrast cardiac CT vs. coronary CT angiography. Quant
Imaging Med Surg 2017;7:326-35.

6. Almutairi A, Al Safran Z, AlZaabi SA, Sun Z. Dual energy CT angiography in peripheral arterial stents: optimal scanning protocols with regard to image quality and radiation dose. Quant Imaging Med Surg 2017;7:520-31.

7. Sun Z, Chaichana T. An investigation of correlation between left coronary bifurcation angle and hemodynamic changes in coronary stenosis by coronary computed tomography angiography-derived computational fluid dynamics. Quant Imaging Med Surg 2017;7:537-48.

8. Hidrovo I, Dey J, Chesal ME, Shumilov D, Bhusal N, Mathis JM. Experimental method and statistical analysis to fit tumor growth model using SPECT/CT imaging: a preclinical study. Quant Imaging Med Surg 2017;7:299-309.

9. Mustapha FA, Bashah FAA, Yassin IM, Fathinul Fikri AS, Nordin AJ, Abdul Razak HR. Estimation of kidneys and urinary bladder doses based on the region of interest in 18fluorine-fluorodeoxyglucose positron emission tomography/computed tomography examination: a preliminary study. Quant Imaging Med Surg 2017;7:310-7.

10. Esposito F, Mamone R, Di Serafino M, Mercogliano C, Vitale V, Vallone G, Oresta P. Diagnostic imaging features of necrotizing enterocolitis: a narrative review. Quant Imaging Med Surg 2017;7:336-44.

11. Additively manufactured custom load-bearing implantable devices: grounds for caution. AMJ 2017;10:694-700.

12. Giannopoulos AA, Steigner ML, George E, Barile M, Hunsaker AR, Rybicki FJ, Mitsouras D. Cardiothoracic applications of 3-dimensional printing. J Thorac Imaging 2016;31:253-72.

13. Zanetti EM, Bignardi C. Mock-up in hip arthroplasty preoperative planning. Acta Bioeng Biomech 2013;15:123-8.

14. Speranza D, Citro D, Padula F, Motyl B, Marcolin F, Cali $\mathrm{M}$, Martorelli $\mathrm{M}$. Additive manufacturing techniques for the reconstruction of 3D fetal faces. Appl Bionics Biomech 2017;2017:9701762.

15. Sun Z, Liu D. A systematic review of clinical value of three-dimensional printing in renal disease. Quant Imaging Med Surg 2018;8:311-25.

16. Chepelev L, Wake N, Ryan J, Althobaity W, Gupta A, Arribas E, Santiago L, Ballard DH, Wang KC, Weadock W, Ionita CN, Mitsouras D, Morris J, Matsumoto J, Christensen A, Liacouras P, Rybicki FJ, Sheikh A and RSNA Special Interest Group for 3D Printing. Radiological Society of North America (RSNA) 3D printing Special Interest Group (SIG): guidelines for medical 3D printing and appropriateness for clinical scenarios. 3D Print Med 2018;4:11. 
17. Chepelev L, Souza C, Althobaity W, Miguel O, Krishna S, Akyuz E, Hodgdon T, Torres C, Wake N, Alexander A, George E, Tang A, Liacouras P, Matsumoto J, Morris J, Christensen A, Mitsouras D, Rybicki F, Sheikh A. Preoperative planning and tracheal stent design in thoracic surgery: a primer for the 2017 Radiological Society of North America (RSNA) hands-on course in 3D printing. 3D Print Med 2017;3:14.

18. Lau IWW, Liu D, Xu L, Fan Z, Sun Z. Clinical value of patient-specific three-dimensional printing of congenital heart disease: Quantitative and qualitative Assessments. PLoS One 2018;13:e0194333.

19. Aldosari S, Squelch A, Sun Z. Patient-specific 3D-printed pulmonary artery model: A preliminary study. Digit Med 2017;3:170-7.

20. Witowski J, Wake N, Grochowska A, Sun Z, Budzynski A, Major P, Popiela TJ, Pedziwiatr M. Investigating accuracy of 3D-printed liver models with computed tomography. Quant Imaging Med Surg 2018. doi: 10.21037/ qims.2018.09.16. [In press].

21. Mankovich NJ, Cheeseman A, Stoker NG. The display of three-dimensional anatomy with stereolithographic models. J Digit Imaging 1990;3:200-3.

22. Winder R, Cooke RS, Gray J, Fannin T, Fegan T. Medical rapid prototyping and $3 \mathrm{D} C \mathrm{CT}$ in the manufacture of custom made cranial titanium plates. J Med Eng Technol 1999;23:26-8.

23. Lau I, Sun Z. Three-dimensional printing in congenital heart disease: A systematic review. J Med Radiat Sci 2018;65:226-36.

24. Costello JP, Olivieri LJ, Krieger A, Thabit O, Marshall MB, Yoo SJ, Kim PC, Jonas RA, Nath DS. Utilizing threedimensional printing technology to assess the feasibility of high-fidelity synthetic ventricular septal defect models for simulation in medical education. World J Pediatr Congenit Heart Surg 2014;5:421-6.

25. Costello JP, Olivieri LJ, Su L, Krieger A, Alfares F, Thabit O, Marshall MB, Yoo SJ, Kim PC, Jonas RA, Nath DS. Incorporating three-dimensional printing into a simulation-based congenital heart disease and critical care training curriculum for resident physicians. Congenit Heart Dis 2015;10:185-90.

26. Jones TW, Seckeler MD. Use of 3D models of vascular rings and slings to improve resident education. Congenit Heart Dis 2017;12:578-82.

27. Lim KH, Loo ZY, Goldie S, Adams J, McMenamin P. Use of 3D-printed models in medical education: A randomized control trial comparing $3 \mathrm{D}$ prints versus cadaveric materials for learning external cardiac anatomy. Anat Sci Educ 2016;9:213-21.

28. Loke YH, Harahsheh AS, Krieger A, Olivieri LJ. Usage of 3D models of tetralogy pf Fallot for medical education: impact on learning congenital heart disease. BMC Med Educ 2017;17:54.

29. White SC, Sedler J, Jones TW, Seckeler M. Utility of three-dimensional models in resident education on simple and complex intracardiac congenital heart defects. Congenit Heart Dis 2018;13:1045-9.

30. Valverde I, Gomez-Ciriza G, Hussain T, Suarez-Mejias C, Velasco-Forte MN, Byrne N, Ordoñez A, Gonzalez-Calle A, Anderson D, Hazekamp MG, Roest AAW, Rivas-Gonzalez J, Uribe S, El-Rassi I, Simpson J, Miller O, Ruiz E, Zabala I, Mendez A, Manso B, Gallego P, Prada F, Cantinotti M, Ait-Ali L, Merino C, Parry A, Poirier N, Greil G, Razavi R, Gomez-Cia T, Hosseinpour AR. Three dimensional printed models for surgical planning of complex congenital heart defects: an international multicenter study. Eur J Cardiothorac Surg 2017;52:1139-48.

31. Ryan J, Plasencia J, Richardson R, Velez D, Nigro JJ, Pophal S, Frakes D. 3D printing for congenital heart disease: a single site's initial three-year experience. 3D Print Med 2018;4:10.

32. Sun $Z$, Lee $S$. A systematic review of $3 \mathrm{D}$ printing in cardiovascular and cerebrovascular diseases. Anatol J Cardiol 2017;17:423-35.

33. Ho D, Squelch A, Sun Z. Modelling of aortic aneurysm and aortic dissection through 3D printing. J Med Radiat Sci 2017;64:10-7.

34. Sun Z, Squelch A. Patient-specific 3D-printed models of aortic aneurysm and aortic dissection. J Med Imaging Health Inf 2017;7:886-9.

35. Hossien A, Gesomino S, Maessen J, Autschbach R. The interactive use of multi-dimensional modeling and 3D printing in preplanning of type A aortic dissection. J Card Surg 2016;31:441-5.

36. Wang H, Liu J, Zheng X, Rong X, Zheng X, Peng H, Silber-Li Z, Li M, Liu L. Three-dimensional virtual surgery models for percutaneous coronary intervention (PCI) optimization strategies. Sci Rep 2015;5:10945.

37. Huang J, Li G, Wang W, Wu K, Le T. 3D printing guiding stent graft fenestration: A novel technique for fenestration in endovascular aneurysm repair. Vascular 2017;25:442-6.

38. Sun Z, Ng CK, Squelch A. Synchrotron radiation computed tomography assessment of calcified plaques and coronary stenosis with different slice thicknesses and beam 
energies on 3D printed coronary models. Quant Imaging Med Surg 2018. doi: 10.21037/qims.2018.09.11. [In press].

39. Perica ER, Sun Z. A systematic review of threedimensional printing in liver disease. J Digit Imaging 2018;31:692-701.

40. Javan R, Zeman M. A prototype educational model for hepatobiliary interventions: unveiling the role of graphic designers in medical 3D printing. J Digit Imaging 2018;31:133-43.

41. Javan R, Herrin D, Tangestanipoor A. Understanding spatially complex segmental and branch anatomy using $3 \mathrm{D}$ printing: liver, lung, prostate, coronary arteries, and circle of willis. Acad Radiol 2016;23:1183-9.

42. Perica E, Sun Z. Patient-specific three-dimensional printing for pre-surgical planning in hepatocellular carcinoma treatment. Quant Imaging Med Surg 2017;7:668-77.

43. Lau I, Squelch A, Wan YL, Wong A, Ducke W, Sun Z. Patient-specific 3D-printed model in delineating brain glioma and surrounding structures in a pediatric patient. Digit Med 2017;3:86-92.

44. Al Jabbari O, Abu Saleh WK, Patel AP, Igo SR, Reardon MJ. Use of three-dimensional models to assist in the resection of malignant cardiac tumors. J Card Surg 2016;31:581-3.

Cite this article as: Sun Z. 3D printing in medicine: current applications and future directions. Quant Imaging Med Surg 2018;8(11):1069-1077. doi: 10.21037/qims.2018.12.06
45. Thawani JP, Singh N, Pisapia JM, Abdullah KG, Parker D, Pukenas BA, Zager EL, Verma R, Brem S. Threedimensional printed modelling of diffuse low-grade gliomas and associated white matter tract anatomy. Neurosurgery 2017;80:635-45.

46. Sun Z, Aldosari S. Three-dimensional printing in medicine: Opportunities for development of optimal CT scanning protocols. AMJ 2018;11:529-32.

47. Abdullah KA, McEntee MF, Reed W, Kench PL. Development of an organ-specific insert phantom generated using a 3D printer for investigations of cardiac computed tomography protocols. J Med Radiat Sci 2018;65:175-83.

48. Aldosari S, Jansen S, Sun Z. Optimization of computed tomography pulmonary angiography protocols using 3D printed model with simulation of pulmonary embolism. Quant Imaging Med Surg 2018. doi: 10.21037/ qims.2018.09.15. [In press].

49. Aldosari S, Jansen S, Sun Z. Patientspecific 3D printed pulmonary artery model with simulation of peripheral pulmonary embolism for developing optimal computed tomography pulmonary angiography protocols. Quant Imaging Med Surg 2018. doi: 10.21037/qims.2018.10.13. [In press]. 